\title{
Computer modelling of the influences of a subsystems' interaction on energetic efficiency of biofuel production systems
}

\author{
Andrzej Wasiak ${ }^{1, *}$, and Olga Orynycz ${ }^{1}$ \\ ${ }^{1}$ Bialystok University of Technology, Dept. of Production Management, Bialystok, Poland
}

\begin{abstract}
Energetic efficiency of biofuel production systems, as well as that of other fuels production systems, can be evaluated on the basis of modified EROEI indicator. In earlier papers, a new definition of the EROEI indicator was introduced. This approach enables the determination of this indicator separately for individual subsystems of a chosen production system, and therefore enables the studies of the influence of every subsystem on the energetic efficiency of the system as a whole. The method has been applied to the analysis of interactions between agricultural, internal transport subsystems, as well as preliminary studies of the effect of industrial subsystem.
\end{abstract}

\section{Introduction}

The problem of the efficiency of energy production has been raised relatively a long time ago mainly in terms of qualitative, philosophical or economic considerations [1]. Much later the quantitative approach was introduced [2]. To the measure of energetic efficiency the name EROEI (or EROI) "Energy Return on Energy Invested" was given. It was defined as a ratio of energy delivered by some production systems to the energy that has to be consumed in order to obtain, and deliver, the above mentioned amount of energy from a given resource. The EROEI is different than thermodynamic efficiency $(\eta)$ of energy converter, which is established as the ratio of energy delivered by the converter to the energy input to that converter. Since some energy is lost (dissipated) during conversion, the values of thermodynamic efficiency are contained within the limits $0 \leq \eta \leq 1$. The EROEI, in contrast, does not consider the input of energy that has to be converted (in fact the input is rather a material resource). What is taken into account is the output of energy from the system, and the subsidiary fluxes of energy being necessary to assure performance of the system. Therefore, this index may assume values from zero to infinity. One may consider the lack of precisely defined calculations procedure as a disadvantage of EROEI. In fact, as indicated in [3], several procedures, and several definitions are used, and some resulting ambiguities have been discussed $[4,5]$. Recently, the definition discussed in [6] was used by present the Authors, in a series of papers [7-10] devoted to the modelling of energy efficiency of agricultural subsystems in the rapeseed biodiesel production system.

It seems clear that the EROEI index can be equally applied to any energy production systems, including fossil fuels, biofuels, solar or wind energy. In recent years EROEI become an important characteristic for alternative energy production systems, because it is possible that in order to gain energy from alternative resources (intended to replace exploitation of fossil ones) one has to consume energy derived from fossil resources. In extreme cases, when the amount of consumed energy would exceed the amount of delivered, such processes would be lacking in sense.

It is also remarkable that fuel production systems are frequently composed of separated subsystems, having different characteristics, but connected to each other by fluxes of masses or energy. Therefore, establishing a way of separating the estimation of energetic efficiency of such subsystems, and the investigation of their interactions determining the total energetic efficiency of the production system become important.

\section{Structure and efficiency of fuel production systems}

The structure of fuel production systems can be classified as serial or parallel composition of components (subsystems) or a combination containing partly serial, partly parallel. Simple examples of such composed systems are shown in Fig. 1 and Fig. 2. The flux of material resources and semi-products are marked with black arrows going down. Fluxes of energy provided to facilitate conversion are plotted as grey arrows.

The EROEI indicator, in this paper is represented by the character $(\varepsilon)$ because certain specific assumptions are introduced for the sake of computations.

\footnotetext{
Corresponding author: a.wasiak@pb.edu.pl
} 


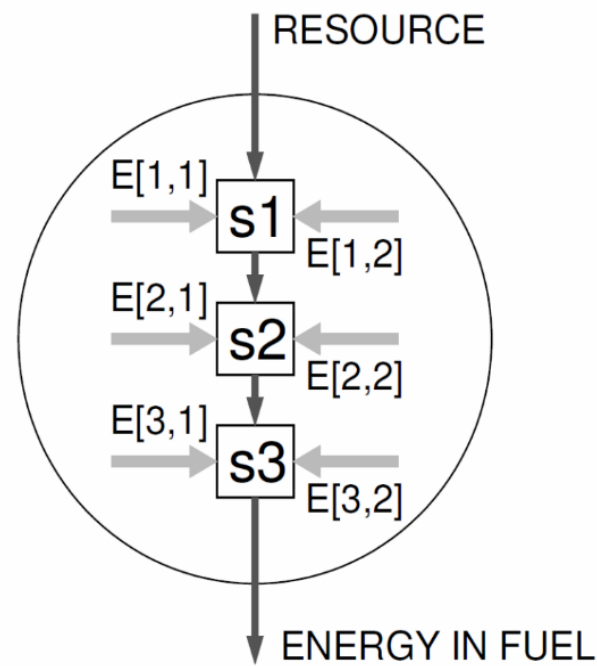

Fig. 1. Fuel production system with serial alignment of subsystems.

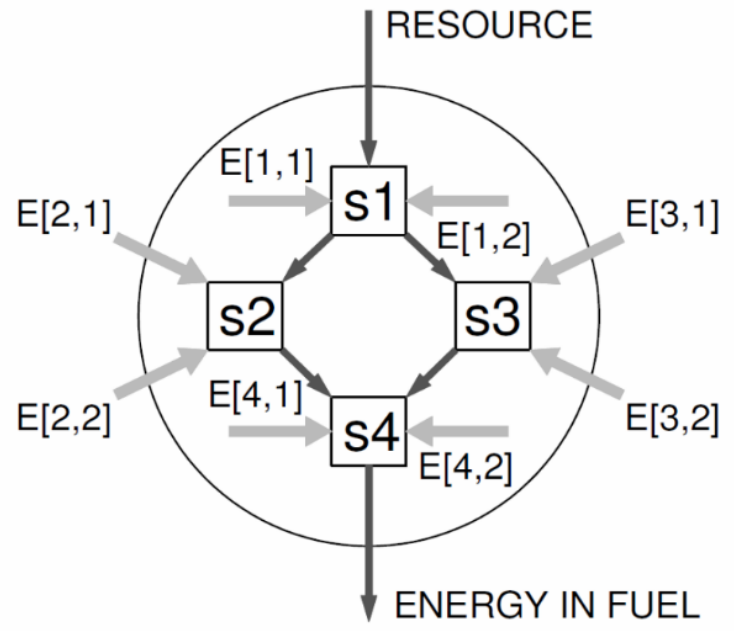

Fig. 2. Fuel production system with combination of serial and parallel alignment of subsystems.

The general definition of energetic efficiency of the production system is accepted as:

$$
\varepsilon_{\text {tot }}=E_{f u e l} / \Sigma E_{i, j}
$$

where index $j$ numbers the energy fluxes going into a given $\mathrm{i}$-th subsystem.

The partial energetic efficiency of the particular subsystem

$$
\varepsilon_{j}=E_{f u e l} / \Sigma E_{i}
$$

In both cases described by Eq. 1, and Eq. 2 the numerator of the expression is the same, and it is the final amount of energy obtained from the system (delivered out of the system). It has to be taken into account that determination of values of partial efficiency (according to Eq. 2) must be related to exactly the same quantity of resource entering the system, although during conversion processes both form and the quantity of related semi-products might be different than the amount of initial resource.

Accepting the above conditions one may easily derive the relation between total, $\varepsilon_{t o t}$, and partial efficiency. Namely:

$$
\varepsilon_{t o t}^{-1}=\sum \varepsilon_{j}^{-1}
$$

It can be seen that for very particular cases, when all the values of partial efficiency in the system are equal, $\varepsilon_{I}=\varepsilon_{2}=\ldots \ldots .=\varepsilon_{I}$, the total efficiency will be represented by:

$$
\varepsilon_{t o t}=\varepsilon_{I} / I
$$

where $I$ is the total number of subsystems.

In the case when individual values of partial energetic efficiency differ from each other; such simple procedures described by Eq. 4 are not valid.

The same formulas can be correspondingly applied to the set of technological operations or processes occurring in the production system. It is important, however, to compute all partial, as well as the final, total efficiency with the same value of total energy obtained from production system from a well-defined, particular amount of resource. In the cases of biofuel production systems, the procedure might refer to particular mass of crops or to particular plantation area.

\section{Numerical computations}

Eq. 4 demonstrates that the addition of subsequent steps of production technology decreases the energetic efficiency of the whole production system. In the case of equal values of partial energetic efficiency of individual subsystems or processes, the simple form of Eq. 4 represents this decrease. When partial contributions are not equal, the general solution cannot be obtained. Consequently, the estimation of the effect for a particular case can be achieved by numerical computations. For the purpose of the present paper, examples of computations have been performed using the Microcal ORIGIN computer program.

For the first case, a system composed of two subsystems is considered. Fig. 3 presents results of computations of energetic efficiency of the whole system, $\varepsilon_{t o t}$, as function of partial efficiency $\varepsilon_{i}$ of both subsystems treated as independent variables. It is seen that the global efficiency of the system is represented by convex surface having local maxima corresponding to equal values of each contributing partial efficiency. The higher are values of both contributing characteristics, the higher is the resulting global energetic efficiency of the system. The maximum observed value of the global efficiency is reduced to half of the values of contributing ones. 


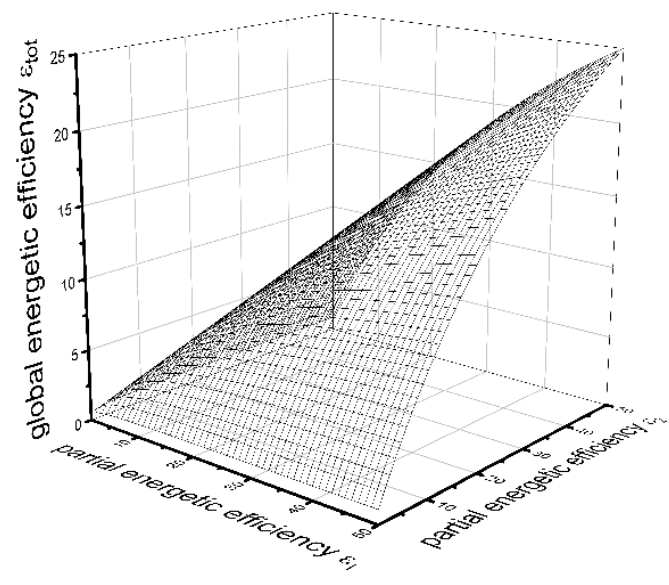

Fig. 3. Energetic efficiency of the production system composed of two subsystems.

Fig. 4 in turn shows the dependence of global energetic efficiency upon the first component efficiency $\left(\varepsilon_{l}\right)$ being considered as an independent variable, for only several chosen values of the second component energetic efficiency, $\varepsilon_{2}$.

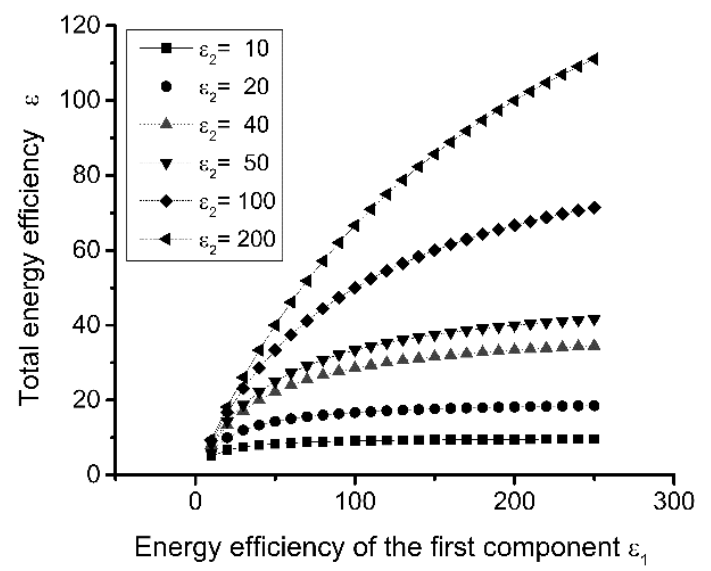

Fig. 4. Energetic efficiency of the production system composed of two subsystems computed for several combinations of subsystem's efficiency.

The dependence of total energetic efficiency of the system appears to be nonlinear function of the efficiency of the first component. The values of energetic efficiency of the system composed within a subsystem of low partial efficiency equal to ten are drastically reduced. Practically for all values of the $\varepsilon_{l}$ being between 50 and 250, the resulting global efficiency is always smaller than ten. In the other extreme case, when the value for $\varepsilon_{2}$, is taken as 200 , the resulting global efficiency is also reduced with respect to the both components, and is always smaller than half of the value for larger component.

The results of calculations for a system composed of three subsystems are shown in Fig. 5. It was assumed that two of the subsystems have the same efficiency, while several values were chosen for the third one.

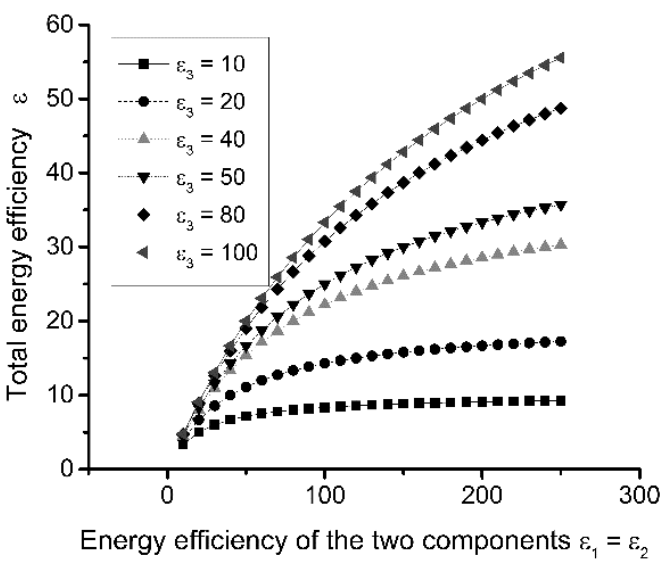

Fig. 5. Energetic efficiency of the production system composed of three subsystems.

This case also shows that global energetic efficiency of the whole production system is greatly reduced with respect to the efficiency of contributing subsystems. The highest reduction is observed when large differences between efficiencies of contributing subsystems occur. It is evident that even both of the two contributing subsystems exhibit high efficiency, the smallest contribution dominates over the remaining, reducing global value to the level even lower than that of the smallest contribution.

Taking into account that any production system is composed of several subsystems, in which several processes are realized, one should expect rather lower global energetic efficiency for the whole production system. Especially dangerous are those subsystems, or processes, which exhibit low energetic efficiency, since they strongly reduce the efficiency of the whole system. In addition, the number of subsystems is the factor strongly reducing the overall efficiency. It is also remarkable, that the highest efficiency is achieved when efficiencies of individual subsystems are close to each other.

\section{Conclusions}

The results of computations presented above demonstrate that computer modelling may serve as an effective tool for analysis of various technical phenomena giving deep insight into relationships between many factors.

The presented results of computations aim to investigate the effects of energetic efficiency of subsystems on the energetic efficiency of a whole system formed from those subsystems, and dedicated to fuel production allows one to formulate several concluding remarks.

First of all, it is evident that combination of several subsystems, each of which consumes energy, strongly reduces the global energetic effectiveness of the whole system. Moreover, the most favourable case is when the 
values of efficiency of each subsystem are equal or close to each other. In the case of large differences, the component of the lowest efficiency dominates reducing total efficiency.

Additionally, reducing the number of subsequent operations (the number of subsystems) leads to an increase in total energetic effectiveness.

Therefore, in designing fuel production systems one should carefully choose composing parts and processes in such a way that the efficiency of each contributing process is as high as possible, and combined processes show similar values of efficiency.

For processes of fuel production it seems necessary to achieve possibly high values of global efficiency of the system, otherwise harvesting energy from particular resources may consume more energy than the process delivers.

Considering alternative energy sources, including alternative fuels, the real replacement of fossil fuels might occur only when the total energetic efficiency of the production system is higher than one. Systems with smaller efficiencies should be avoided. Due to the existence of some rules of economic subventions given to alternative energy producers, one might expect the existence of a discrepancy between the efficient production of energy and economic profits as a result of that production. This point requires separate studies.

The proposed definition and the method of calculation of energetic efficiency of energy production systems offers the possibility of studying how individual components affect the global energetic efficiency of the whole system.

The elaborated rule of additivity for reciprocals of partial efficiencies of subsystems that results in assumed methodology opens new functionality for energetic efficiency indicators.

The research has been performed under the financial support from the Bialystok University of Technology. The Statutory Research Project S/WZ/1/2015 was financed from the scientific grant received from the Ministry of Science and Higher Education (MNiSW).

\section{References}

1. T. Martinez-Allier, R. Muradian, Handbook of Ecological Economics (Edward Elgar Publ., (2015)

2. C.J. Cleveland, R. Costanza, C.A. Hall, R. Kaufmann, Science, 225 (1984)

3. D.J. Murphy, C.A. Hall, Ann. N.Y. Acad. Sci., 1185 (2010)

4. Y. Zhang, L.M. Colosi, Energy Policy, 57 (2013)

5. D.J. Murphy, C.A.S. Hall, M. Dale, C. Cleveland, Sustainability, 3 (2011)

6. W.F. Pickard, Proceedings of the IEEE, 102, 8 (2014)

7. A. Wasiak, O. Orynycz, IEEE International Energy Conference: ENERGYCON'2014, Dubrovnik, Croatia, (2014)

8. A. Wasiak, O.Orynycz, Agriculture and Agricultural Science Procedia, 7 (2015)

9. O. Orynycz, A. Wasiak, Appl. Computer Sci., 10, 2 (2014).

10. A. Wasiak, O. Orynycz, Appl. Computer Sci., 11, 1 (2015). 